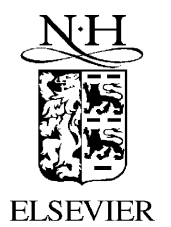

ELSEVIER

\title{
On hexagonal gravity water waves
}

\author{
David P. Nicholls* \\ School of Mathematics, Institute of Technology, University of Minnesota, 127 Vincent Hall, \\ 206 Church Street S.E., Minneapolis, MN 55455, USA
}

Received 1 October 2000; accepted 30 October 2000

\begin{abstract}
In this paper we produce numerical, genuinely three-dimensional, hexagonal traveling wave solutions of the Euler equations for water waves using a surface integral formulation derived by Craig and Sulem. These calculations are free from the requirements of either long wavelength or two-dimensionality, both of which are crucial to the KdV and KP scaling regimes, and we produce hexagonal traveling waves of not only small but also moderate amplitude. (C) 2001 IMACS. Published by Elsevier Science B.V. All rights reserved.
\end{abstract}

Keywords: Water waves; Hexagonal traveling waves

\section{Introduction}

One of the fundamental problems of fluid mechanics is to describe the motion of waves on the surface of a body of water. The experiments of Russell [29] on progressing water waves inspired the work of Airy [3], Stokes [33], and Boussinesq [7,8] who produced solutions to model equations (of the governing Euler equations) based on certain scaling assumptions. These assumptions include small amplitude, long wavelength, and two-dimensionality of the wave form (for this paper a fluid has one vertical dimension and $(n-1)$ horizontal dimensions). This work has been the foundation of much of the progress in the field of water waves in the past 150 years [22,34].

The understanding of genuinely three-dimensional waves is much less complete than that of twodimensional waves, although perturbative and numerical approaches have yielded results in several regimes (see [20]). In the present paper we study genuinely three-dimensional waves which progress steadily without change of form (traveling waves). In particular, our goal has been to model and explain the traveling patterns noticed in the experimental work of Su [35], Su et al. [30], and Hammack et al. $[19,20]$. Bryant [9,10], Saffman and Yuen [36,37], and Meiron et al. [24] have all calculated waves using a Fourier approach. Hammack et al. $[19,20]$ remarked on the tendency of such three-dimensional wave

\footnotetext{
* Fax: +1-612-626-2017.

E-mail address: nicholls@math.umn.edu (D.P. Nicholls).
}

0378-4754/01/\$20.00 @ 2001 IMACS. Published by Elsevier Science B.V. All rights reserved.

PII: S 0378-4754(00)00296-2 
patterns to take on hexagonal form, and they matched their experiments to exact traveling wave solutions of the KP equation [21] via a surface-fitting algorithm (see also [32]). Finally, Milewski and Keller [23] found numerical solutions of a certain model of the Euler equations using Fourier spectral methods.

In this paper we produce numerical, genuinely three-dimensional, hexagonal traveling wave solutions of the Euler equations for water waves using a surface integral formulation derived by Craig and Sulem [14] and a numerical implementation described in [27]. These calculations are free from the requirements of either long wavelength or two-dimensionality, both of which are crucial to the KdV and KP scaling regimes, and we produce hexagonal traveling waves of not only small, but also moderate amplitude. We note that in this work we consider flows without surface tension, however, inclusion of such effects would pose no significant obstacle to our method. In Section 2 we discuss the equations of motion of water waves (the Euler equations), Section 3 describes our method for finding hexagonal waves, and in Section 4 we present our numerical results of hexagonal waves in shallow water, water of moderate depth, and water of infinite depth.

\section{Equations of motion}

Consider the fluid domain

$$
S_{h, \eta}=\left\{(x, y) \in \mathbb{R}^{n-1} \times \mathbb{R} \mid-h<y<\eta(x, t)\right\}
$$

of depth $h$ (possibly infinite) bounded above by the free surface $\eta(x, t)$. The equations of motion for an $n$-dimensional ideal fluid occupying $S_{h, \eta}$ under the influence of gravity are $[1,22]$

$$
\begin{aligned}
& \Delta \varphi=0 \quad \text { in } S_{h, \eta} \\
& \partial_{y} \varphi=0 \quad \text { at } y=-h \\
& \partial_{t} \eta+\nabla_{x} \varphi \cdot \nabla_{x} \eta-\partial_{y} \varphi=0 \quad \text { at } y=\eta \\
& \partial_{t} \varphi+\frac{1}{2}|\nabla \varphi|^{2}+g \eta=0 \quad \text { at } y=\eta
\end{aligned}
$$

where $\varphi(x, y, t)$ is the velocity potential and $g$ is the constant gravity. These equations can be restated entirely at the free surface of the fluid [14,38] in terms of the surface quantities $\eta(x, t)$ and $\xi(x, t)=$ $\varphi(x, \eta(x, t), t)$, provided one allows the introduction of the Dirichlet-Neumann operator (DNO) which maps Dirichlet data to Neumann data

$$
G(\eta) \xi=\left.(\nabla \varphi)\right|_{y=\eta} \cdot N_{\eta}, \quad N_{\eta}=\left(-\nabla_{x} \eta, 1\right)^{\mathrm{T}}
$$

Furthermore, Zakharov [38] has established that the water wave equations (1) are a Hamiltonian system with canonical variables $\eta, \xi$ and Hamiltonian [14]

$$
H=\frac{1}{2} \int \xi G(\eta) \xi+g \eta^{2} \mathrm{~d} x
$$

By setting $u=(\eta, \xi)^{\mathrm{T}}$ and $J=\left(\begin{array}{cc}0 & -1 \\ 1 & 0\end{array}\right)$, Eq. (1) can be restated as

$$
\partial_{t} u=J \delta_{u} H
$$


In order to study traveling wave solutions we switch to a coordinate reference frame moving with speed $c \in \mathbb{R}^{n-1}$ and seek steady solutions. The equations for such traveling wave solutions are given by $[26,27]$

$$
\begin{aligned}
& g \eta+\left[c \cdot \nabla_{x}\right] \xi+\frac{1}{2\left(1+\left|\nabla_{x} \eta\right|^{2}\right)}\left[\left|\nabla_{x} \xi\right|^{2}-(G(\eta) \xi)^{2}-2(G(\eta) \xi) \nabla_{x} \xi \cdot \nabla_{x} \eta+\left|\nabla_{x} \xi\right|^{2}\left|\nabla_{x} \eta\right|^{2}\right. \\
& \left.\quad-\left(\nabla_{x} \xi \cdot \nabla_{x} \eta\right)^{2}\right]=0 \\
& -\left[c \cdot \nabla_{x}\right] \eta+G(\eta) \xi=0
\end{aligned}
$$

For notational convenience we will often refer to (3) as $F(u, c)=0$.

Analyticity properties of the DNO have been studied by Coifman and Meyer [12], Craig et al. [15], Craig and Nicholls [13], and Nicholls and Reitich [28]. These properties justify the expansion of the DNO in powers of $\eta$ about zero (quiescent water)

$$
G(\eta) \xi=\sum_{n=0}^{\infty} G_{n}(\eta) \xi
$$

where $G_{n}(\varepsilon \eta)=\varepsilon^{n} G_{n}(\eta)$. We note for future reference that at order zero one can explicitly compute the DNO, $G_{0}$, as the Fourier multiplier [14]

$$
G_{0} \xi=|D| \tanh (h|D|) \xi=\int|k| \tanh (h|k|) \hat{\xi}(k) \mathrm{d} k
$$

Various methods have been proposed for computing DNO (or equivalent quantities) based on Fourier series (see e.g. [17]), boundary integrals (see e.g. [4-6]), operator expansions [14,26,27,31], and Fourier series with domain flattening changes of variables [28]. The computations presented here are based on operator expansions [14] and have given us spectrally accurate results within the parameter ranges given in this paper. Please see [28] for more details on the computation of DNO.

\section{A method for finding hexagonal traveling waves}

With spectral numerical methods in mind we assume periodic boundary conditions for $x$ (with respect to some lattice $\Gamma$ in $\mathbb{R}^{n-1}$ ) in Eq. (3). In order to find hexagonal traveling wave solutions we note that one always has the trivial solution $u=0$ for any $c$, and look for branches of solutions bifurcating from this trivial branch. Bifurcation theory states that a bifurcation can only occur when the linearized operator is singular; for water waves this concerns the singularity of the operator

$$
A(c)=\left(\begin{array}{cc}
g & c \cdot \nabla_{x} \\
-c \cdot \nabla_{x} & G_{0}
\end{array}\right)
$$

which acts on function pairs $(\eta, \xi)^{\mathrm{T}}$. In Fourier space the operator $A(c)$ is block $2 \times 2$ diagonalized [27] and the question of singularity can be reduced to the singularity of these blocks which is measured by the $k$ th determinant

$$
\Delta(c, k)=g|k| \tanh (h|k|)-(c \cdot k)^{2}
$$



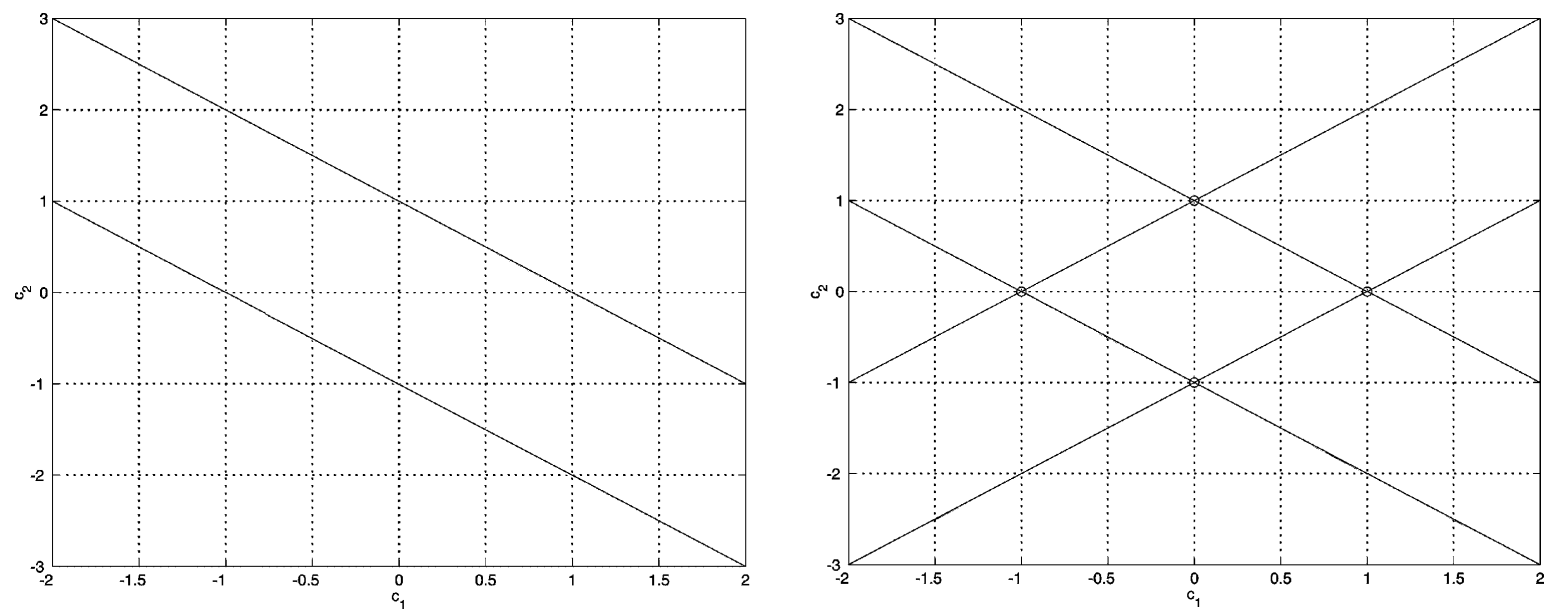

Fig. 1. The zero sets of $\Delta$ (for one and two wavenumbers).

The corresponding null space is spanned by

$$
\psi_{1}(x ; k)=\left(\begin{array}{c}
c \cdot k \cos (k \cdot x) \\
-g \sin (k \cdot x)
\end{array}\right), \quad \psi_{2}(x ; k)=\left(\begin{array}{c}
c \cdot k \sin (k \cdot x) \\
g \cos (k \cdot x)
\end{array}\right)
$$

In $n=2$ dimensions, given a wavenumber $k \in \Gamma^{\prime}$ there will be a unique (up to sign) speed $c_{k} \in \mathbb{R}$ so that $\Delta=0$. Therefore, emanating from $c_{k}$ will be a surface of non-trivial solutions which, for small amplitudes, look like $\alpha \psi_{1}(x ; k)+\beta \psi_{2}(x ; k)$, and are parameterized by $(\alpha, \beta)$; please see [13] for a further discussion of existence of such solution surfaces. We note that the solutions on these surfaces are simply Stokes waves.

In $n=3$ dimensions, given a wavenumber $k \in \Gamma^{\prime}$ there is an entire line (up to symmetry) of speeds $c \in \mathbb{R}^{2}$ so that $\Delta=0$ (see Fig. 1). From a generic point along this line one finds a surface of solutions which is a two-dimensional pattern (a Stokes wave) in a rotated set of coordinates. However, if a second wavenumber $\tilde{k} \in \Gamma^{\prime}$ is chosen, in general (as long as $k$ and $\tilde{k}$ are linearly independent) one can find an intersection of lines in the $c$ plane resulting in a speed $c^{*}$ so that $\Delta\left(c^{*}, k\right)=0$ and $\Delta\left(c^{*}, \tilde{k}\right)=0$ (see Fig. 1). From such a point $c^{*}$ one finds a surface of solutions which, for small amplitudes, have the character $\alpha \psi_{1}(c ; k)+\beta \psi_{1}(c ; \tilde{k})+\gamma \psi_{2}(c ; k)+\delta \psi_{2}(c ; \tilde{k})$, and is parameterized by two parameters. The character of these traveling wave solutions will be altered by the fundamental domain describing the period of the solution, the depth of the fluid, and the relative magnitudes of $\alpha, \beta, \gamma, \delta$. Without loss of generality we fix a peak of our wave-field at the origin which implies that $\gamma=\delta=0$. The central goal of this research is to demonstrate that emanating from the bifurcation point $c^{*}$ is a bifurcation surface of solutions. In an appropriate regime, this surface of solutions consists of wave patterns which are hexagonal in nature.

\section{Numerical results}

To produce numerical solutions of Eq. (3) with periodic boundary conditions it is natural to utilize a spectral Fourier collocation method [11,18]. This procedure transforms the equations $F(u, c)=0$ into 
the finite set of $N$ equations $F_{N}\left(u_{N}, c\right)=0$ with solution $u_{N}$, a trigonometric polynomial which coincides with the exact solution at the $N$ equally spaced collocation points. Using a numerical continuation method [2], one traces out an entire solution branch given a single solution on that branch. The trivial branch of solutions $(u=0, c)$ provides an infinite number of such initial points for our purposes. Furthermore, these continuation techniques can locate bifurcation points and step onto new branches emanating from them. In our case we know explicitly the location of such points along the trivial branch (the set of $c$ such that $\Delta=0)$, and the direction in which to step from these points $\left(\psi_{1}\right.$ and $\left.\psi_{2}\right)$.

In addition to this zeroth order information, which the analysis of the linearized operator about the trivial solution provides, Craig and Nicholls [25] have computed higher order information about solution branches in the setting of three-dimensional waves with a four-dimensional null space $(\gamma=\delta=0)$. Their results are that

$$
c=c^{*}+\varepsilon^{2} c^{(2)}+\mathcal{O}\left(\varepsilon^{3}\right)
$$

where $c^{*}$ satisfies

$$
\Delta\left(c^{*}, k\right)=0, \quad \Delta\left(c^{*}, \tilde{k}\right)=0
$$

(see Section 3) and

$$
\left(\begin{array}{l}
\eta \\
\xi
\end{array}\right)=\varepsilon\left(\begin{array}{l}
\eta^{(1)} \\
\xi^{(1)}
\end{array}\right)+\varepsilon^{2}\left(\begin{array}{l}
\eta^{(2)} \\
\xi^{(2)}
\end{array}\right)+\mathcal{O}\left(\varepsilon^{3}\right)
$$

where

$$
\left(\begin{array}{l}
\eta^{(1)} \\
\xi^{(1)}
\end{array}\right)=a_{1} \psi_{1}\left(c^{*}, k\right)+a_{2} \psi_{2}\left(c^{*}, \tilde{k}\right)
$$

In particular, the perturbation analysis of [25] provides formulae for $c^{(2)}, \eta^{(2)}$, and $\xi^{(2)}$ so that highly accurate steps can be taken onto the primary bifurcation branches. This technique was extensively used in our numerical work and allowed for computations to proceed significantly higher up on the branch than could be realized with the zeroth order information given by the simpler analysis.

Symmetric hexagonal patterns can be generated by choosing a fundamental domain of high aspect ratio and then selecting two symmetric wavenumbers from among the resulting conjugate lattice. In the following calculations the fundamental domain (in this case a rectangle) is generated by the vectors

$$
\left(\begin{array}{l}
1 \\
0
\end{array}\right) \quad\left(\begin{array}{l}
R \cos \Theta \\
R \sin \Theta
\end{array}\right)
$$

where $R=11$ and $\Theta=\pi / 2$. The resulting conjugate lattice $\Gamma^{\prime}$ is generated by the vectors

$$
v_{1}=\left(\begin{array}{c}
2 \pi \\
0
\end{array}\right), \quad v_{2}=\left(\begin{array}{c}
0 \\
\frac{2 \pi}{11}
\end{array}\right)
$$

and thus a choice of symmetric wavenumbers is

$$
k=v_{1}+v_{2}=\left(\begin{array}{c}
2 \pi \\
2 \pi \\
\hline 11
\end{array}\right), \quad \tilde{k}=v_{1}-v_{2}=\left(\begin{array}{c}
2 \pi \\
-\frac{2 \pi}{11}
\end{array}\right)
$$


Based on these wavenumbers and the depth of the fluid $h$, a wave speed $c^{*}$ is selected such that Eq. (5) is satisfied. Using this and the information from Eqs. (4) and (6) an excellent approximation to a solution on the bifurcation surface is obtained which is then further refined with Newton's method. This converged solution is then used as the initial solution in a numerical continuation method.

The experiment outlined above was performed with four fluid depths $h_{1}=\frac{1}{100}, h_{2}=\frac{1}{10}, h_{3}=1$, and $h_{4}=\infty$ on the periodic domain $[0,1] \times[0,11]$ with $g=1$. The spatial discretizations were performed with $N_{1}=N_{2}=32$ equally spaced collocation points in the $x_{1}$ and $x_{2}$ directions, respectively. Also, $m=4$ terms in the expansion of the DNO were considered. The plots of the tallest (among the ones we computed), and thus most nonlinear, solutions are presented in Figs. 2-5. A table summarizing some important measurements of each wave are given in Table 1 . In this table, the momentum, $P$, is given by

$$
P=\int_{P(\Gamma)}\left(\nabla_{x} \eta\right) \xi \mathrm{d} x
$$

and the Stokes number, $S$, is given by

$$
S=\frac{\text { amplitude }^{3}}{\text { Volume (fluid domain) }}=\frac{a^{3}}{11 h} .
$$

Several qualitative statements can be made concerning the appearance of these traveling wave forms. The first is that the solutions for shallow depth display flatter and more shallow troughs, and more sharply peaked crests. Clearly this is due to effects from the bottom, but may indicate that these solutions are "more nonlinear" (i.e. further up along the branch). We also note that the shapes of the hexagons differ as the depth is varied. In the case of waves in deep water, the "major" crests, traveling in the direction of propagation, are much longer and the "minor" crests, connecting the major crests to one another, are quite small. On the other hand, in the shallow depth case, both major and minor crests have significant extent. Finally, the waves in the case of $h=1$ are nearly indistinguishable from the case of $h=\infty$ indicating that "deep water traveling waves" are apparent at depths typically not considered deep.
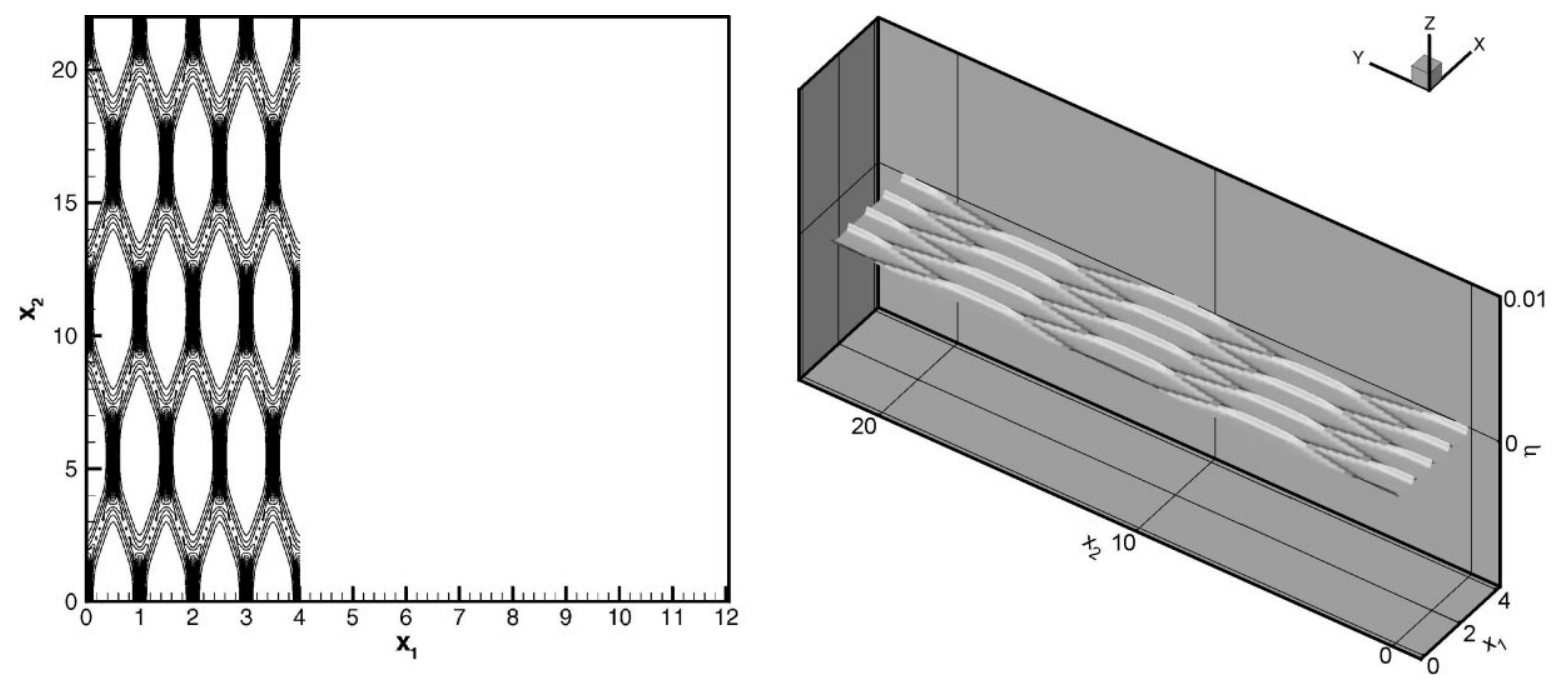

Fig. 2. Contour and surface plots of a hexagonal wave $\left(h=\frac{1}{100}\right)$. 

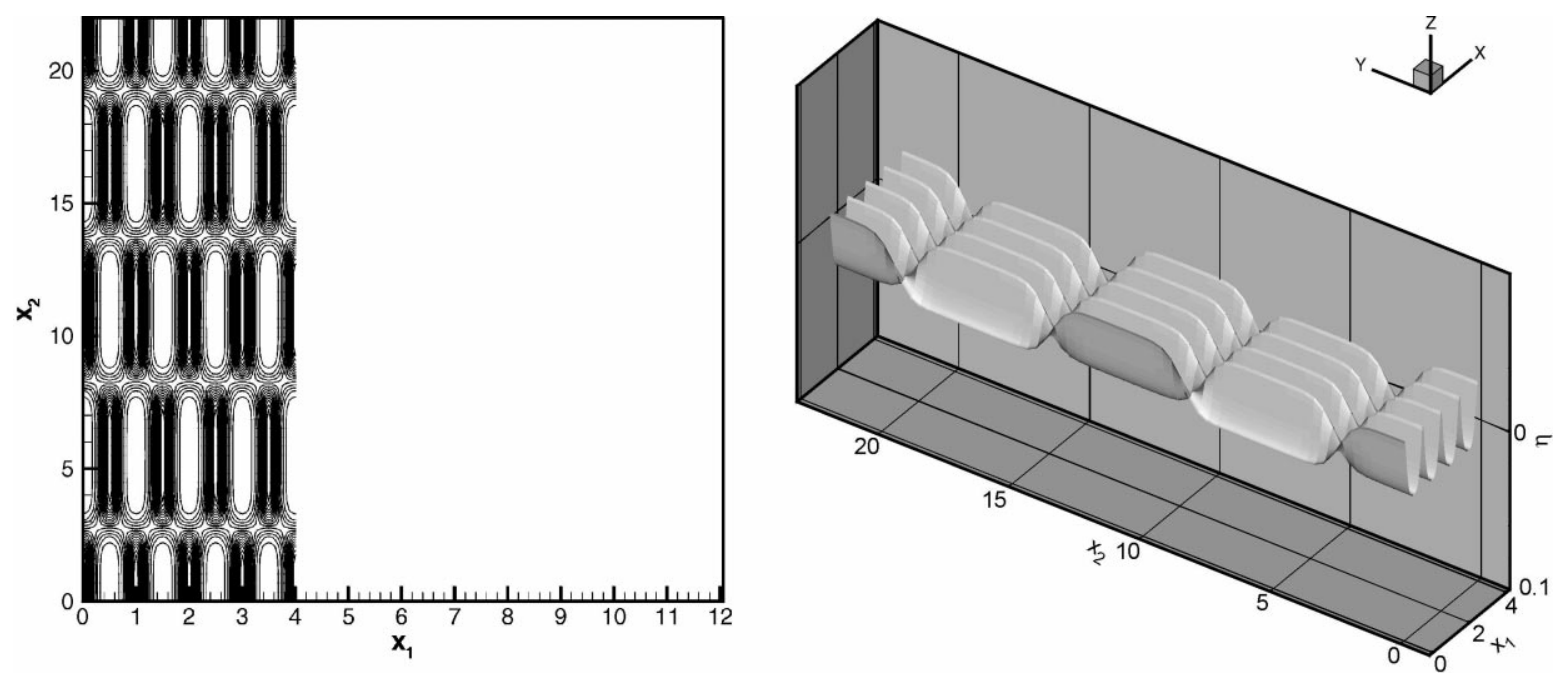

Fig. 3. Contour and surface plots of a hexagonal wave $\left(h=\frac{1}{10}\right)$.

In conclusion we note that these wave shapes are only a few among a large number of interesting configurations that can be computed using our approach. In fact, it is the goal of a forthcoming publication [25] to generate and analyze traveling wave solutions in three dimensions that have unsymmetric hexagonal, unsymmetric crescent, and symmetric crescent shapes (please see $[16,30,35])$. We will present studies of hexagonal wave patterns, crescent-shaped wave patterns in resonant regimes, and model calculations of the skew wave patterns observed by Su [35].
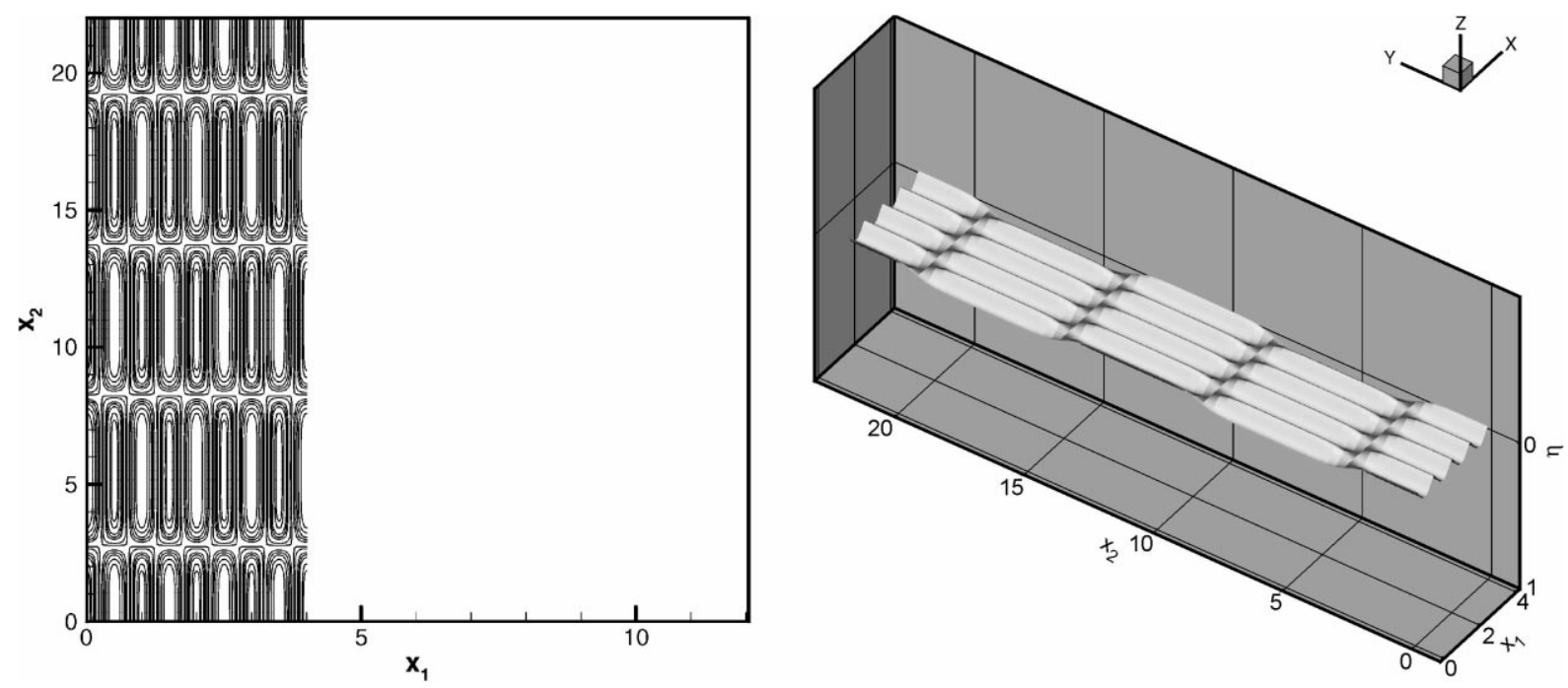

Fig. 4. Contour and surface plots of a hexagonal wave $(h=1)$. 

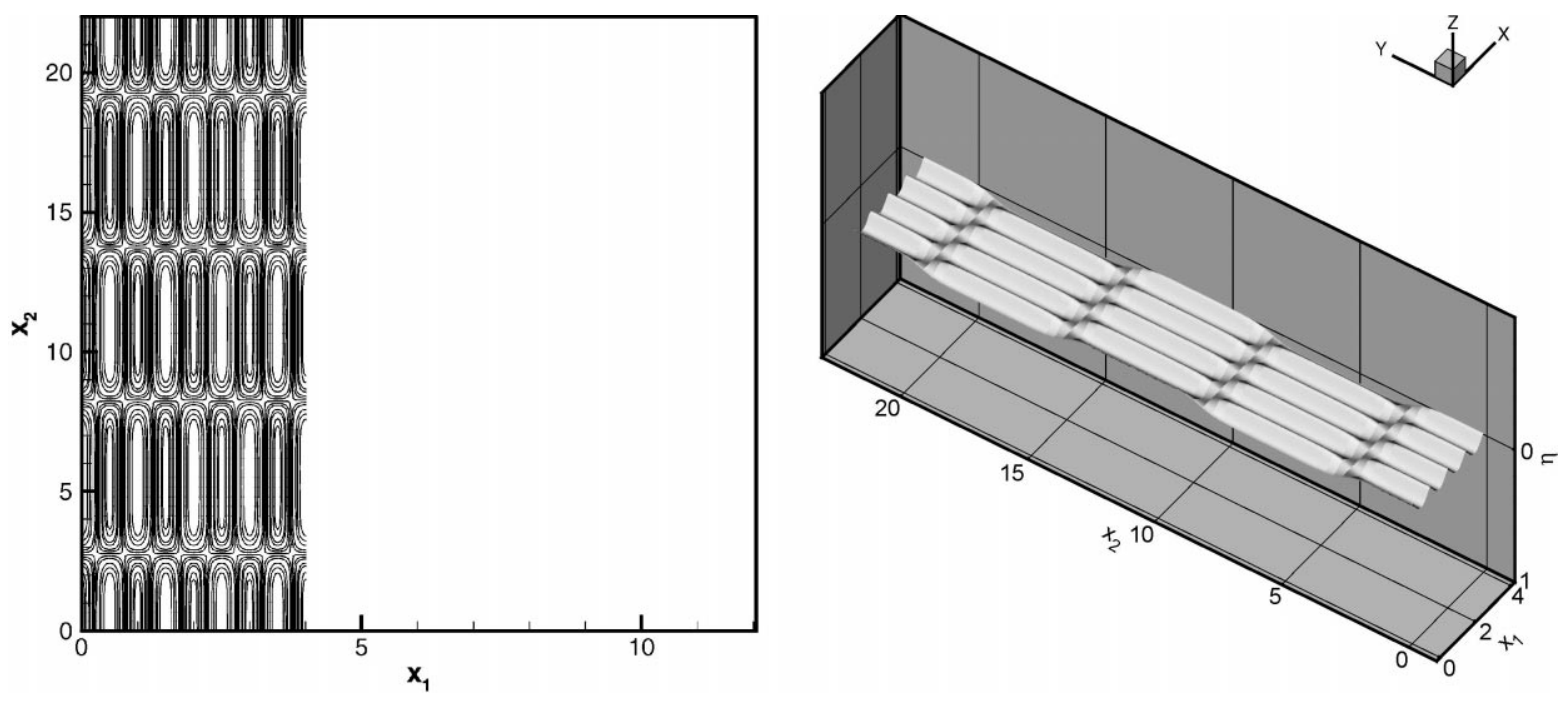

Fig. 5. Contour and surface plots of a hexagonal wave $(h=\infty)$.

Table 1

Data for hexagonal traveling waves

\begin{tabular}{lllllll}
\hline$h$ & $c^{*}$ & $\varepsilon$ & $|\eta|_{L^{\infty}}$ & $|\eta|_{C^{1}}$ & $P$ & $S$ \\
\hline 0.01 & $(0.1013,0)$ & 0.0001 & 0.0003413 & 0.004868 & $3.769 \times 10^{-7}$ & $3.614 \times 10^{-10}$ \\
0.1 & $(0.3131,0)$ & 0.005 & 0.02638 & 0.1875 & 0.002989 & $1.668 \times 10^{-5}$ \\
1 & $(0.4131,0)$ & 0.011 & 0.04825 & 0.2758 & 0.01089 & $1.021 \times 10^{-5}$ \\
$\infty$ & $(0.4131,0)$ & 0.011 & 0.04825 & 0.2758 & 0.01089 & 0 \\
\hline
\end{tabular}

\section{References}

[1] D.J. Acheson, Elementary Fluid Dynamics, Clarendon Press/Oxford University Press, New York, 1990.

[2] E.L. Allgower, K. Georg, Numerical Continuation Methods: An Introduction, Springer, Berlin, 1990.

[3] G.B. Airy, Tides and waves, Encyclopedia Metropolitana 5 (1845) 241-396.

[4] J.T. Beale, T.Y. Hou, J. Lowengrub, Convergence of a boundary integral method for water waves, SIAM J. Numer. Anal. 33 (5) (1996) 1797-1843.

[5] J.T. Beale, T.Y. Hou, J. Lowengrub, Stability of boundary integral methods for water waves, in: Nonlinear Evolutionary Partial Differential Equations (Beijing, 1993), American Mathematical Society, Providence, RI, 1997, pp. $107-127$.

[6] G.R. Baker, D.I. Meiron, S.A. Orszag, Generalized vortex methods for free-surface flow problems, J. Fluid Mech. 123 (1982) 477-501.

[7] M.J. Boussinesq, Théorie des ondes et des remous qui se propegeant le long d'un canal rectangulaire horizontal, en communiquant au liquide contenu dans ce canal des vitesses sensiblement pareilles de la surface au fond, J. Math. Pures Appl. 17 (1872) 55-108.

[8] M.J. Boussinesq, Essai sur la théorie des eaux courantes. Mém. Préséntes par divers Savants à L'Acad, Sci. Inst. France (séries 2) 23 (1877) 1-680.

[9] P.J. Bryant, Two-dimensional periodic permanent waves in shallow water, J. Fluid Mech. 115 (1982) 525-532.

[10] P.J. Bryant, Doubly periodic progressive permanent waves in deep water, J. Fluid Mech. 161 (1985) $27-42$.

[11] C. Canuto, M.Y. Hussaini, A. Quarteroni, T.A. Zang, Spectral Methods in Fluid Dynamics, Springer, New York, 1988. 
[12] R. Coifman, Y. Meyer, Nonlinear harmonic analysis and analytic dependence, in: Pseudodifferential Operators and Applications (Notre Dame, 1984), American Mathematical Society, Providence, RI, 1985, pp. 71-78.

[13] W. Craig, D.P. Nicholls, Traveling two and three dimensional capillary gravity water waves, SIAM: Math. Anal. 32 (2) (2000) 323-359.

[14] W. Craig, C. Sulem, Numerical simulation of gravity waves, J. Comput. Phys. 108 (1993) 73+.

[15] W. Craig, U. Schanz, C. Sulem, The modulation regime of three-dimensional water waves and the Davey-Stewartson system, Ann. Inst. Henri Poincaré 14 (1997) 615-667.

[16] F. Dias, C. Kharif, Nonlinear gravity and capillary-gravity waves, in: Annual review of fluid mechanics, Annual Reviews, Vol. 31, Palo Alto, CA, 1999, pp. 301-346.

[17] D.G. Dommermuth, D.K.P. Yue, A high-order spectral method for the study of nonlinear gravity waves, J. Fluid Mech. 184 (1987) 267-288.

[18] D. Gottlieb, S.A. Orszag, Numerical analysis of spectral methods: theory and applications, Society for Industrial and Applied Mathematics, Philadelphia, PA, 1977. CBMS-NSF Regional Conference Series in Applied Mathematics, No. 26.

[19] J. Hammack, D. McCallister, N. Scheffner, H. Segur, Two-dimensional periodic waves in shallow water. Part 2. Asymmetric waves, J. Fluid Mech. 285 (1995) 95+.

[20] J. Hammack, N. Scheffner, H. Segur, Two-dimensional periodic waves in shallow water, J. Fluid Mech. 209 (1989) 567+.

[21] B. Kadomtsev, V. Petviashvili, On the stability of solitary waves in weakly dispersing media, Sov. Phys. Dokl. 15 (1970) 539-541.

[22] H. Lamb, Hydrodynamics, 6th Edition (foreword by R.A. Caflisch), Cambridge University Press, Cambridge, 1993.

[23] P.A. Milewski, J.B. Keller, Three-dimensional water waves, Stud. Appl. Math. 97 (2) (1996) 149-166.

[24] D. Meiron, P. Saffman, H. Yuen, Calculation of steady three-dimensional deep-water waves, J. Fluid Mech. 124 (1982) $109+$.

[25] W. Craig, D.P. Nicholls, Traveling gravity water waves in two and three dimensions, Preprint, 1999.

[26] D.P. Nicholls, Traveling gravity water waves in two and three dimensions, Ph.D. Thesis, Brown University, 1998.

[27] D.P. Nicholls, Traveling water waves: spectral continuation methods with parallel implementation, J. Comput. Phys. 143 (1) (1998) 224-240.

[28] D.P. Nicholls, F. Reitich, A new approach to analyticity of Dirichlet-Neumann operators, submitted.

[29] J.S. Russell, Report on waves, in: Report of the Fourteenth Meeting of the British Association for the Advancement of Science, 1844, pp. 311-390.

[30] M.-Y. Su, M. Bergin, P. Marler, R. Myrick, Experiments on nonlinear instabilities and evolution of steep gravity-wave trains, J. Fluid Mech. 124 (1982) 45+.

[31] U. Schanz, On the evolution of gravity-capillary waves in three dimensions, Ph.D. Thesis, University of Toronto, Toronto, 1997.

[32] H. Segur, A. Finkel, An analytical model of periodic waves in shallow water, Stud. Appl. Math. 73 (3) (1985) $183-220$.

[33] G.G. Stokes, On the theory of oscillatory waves, Trans. Cambridge Philos. Soc. 8 (1847) 441-473.

[34] J.J. Stoker, Water Waves, Wiley, New York, 1992. The Mathematical Theory with Applications, Reprint of the 1957 Original, Wiley/Interscience, New York.

[35] M.-Y. Su, Three-dimensional deep-water waves. Part I. Experimental measurement of skew and symmetric wave patterns, J. Fluid Mech. 124 (1982) 73+.

[36] P.G. Saffman, H.C. Yuen, A new type of three-dimensional deep-water wave of permanent form, J. Fluid Mech. 101 (4) (1980) 797-808.

[37] P.G. Saffman, H.C. Yuen, Three-dimensional waves on deep water, in: Advances in Nonlinear Waves, Vol. II, Pitman, Boston, MA, 1985, pp. 1-30.

[38] V. Zakharov, Stability of periodic waves of finite amplitude on the surface of a deep fluid, J. Appl. Mech. Tech. Phys. 9 (1968) $190+$. 